\title{
ERCP during the COVID-19 epidemic
}

This is a reply to the letter by Dr. Douglas K. Pleskow and colleagues.

During the COVID-19 outbreak in Wuhan city, from 20 January to 31 March 2020, a total of 16 endoscopic retrograde cholangiopancreatographies (ERCPs) were performed in Zhongnan Hospital, among which one was performed in a patient with pyogenic cholangitis who had been diagnosed as having COVID-19 [1].

An isolation ward was specially set for this COVID-19 patient. He was transferred to the endoscopy center via a preset route with the use of an $\mathrm{N} 95$ mask all the way. Within half an hour, the ERCP procedure, including catheterization, stone extraction, and endoscopic nasobiliary drainage (ENBD), was completed. On the operation day, only the necessary medical staff were allowed to remain in the endoscopy center, in order to reduce the exposed population. To avoid the potential risk of droplet and aerosol transmission, protection at biosafety level 3, including a positive pressure ventilation hood, was used by all medical staff (-Fig. 1a,b).

After the operation, the used duodenoscope was immediately packed into a double-layer medical waste-bag and sent to the disinfection room [2]. All used accessories and produced medical waste were also immediately packed into double-layer medical waste-bags and were treated accordingly. Endoscopic equipment and radiographic machinery in the operation room were disinfected by spraying with $75 \%$ alcohol. Chlorine-containing detergent was used for floor cleaning and an ozone system was used for air cleaning. Until the above disinfection procedure had been completed, staff were allowed to take off their personal protective equipment in a designated buffer zone.

For patients in whom COVID-19 had been excluded, biosafety level 2 protection was used by medical staff ( $\triangleright$ Fig. 1c).

Because coronaviruses, including SARSCoV-2, can be inactivated by many com-
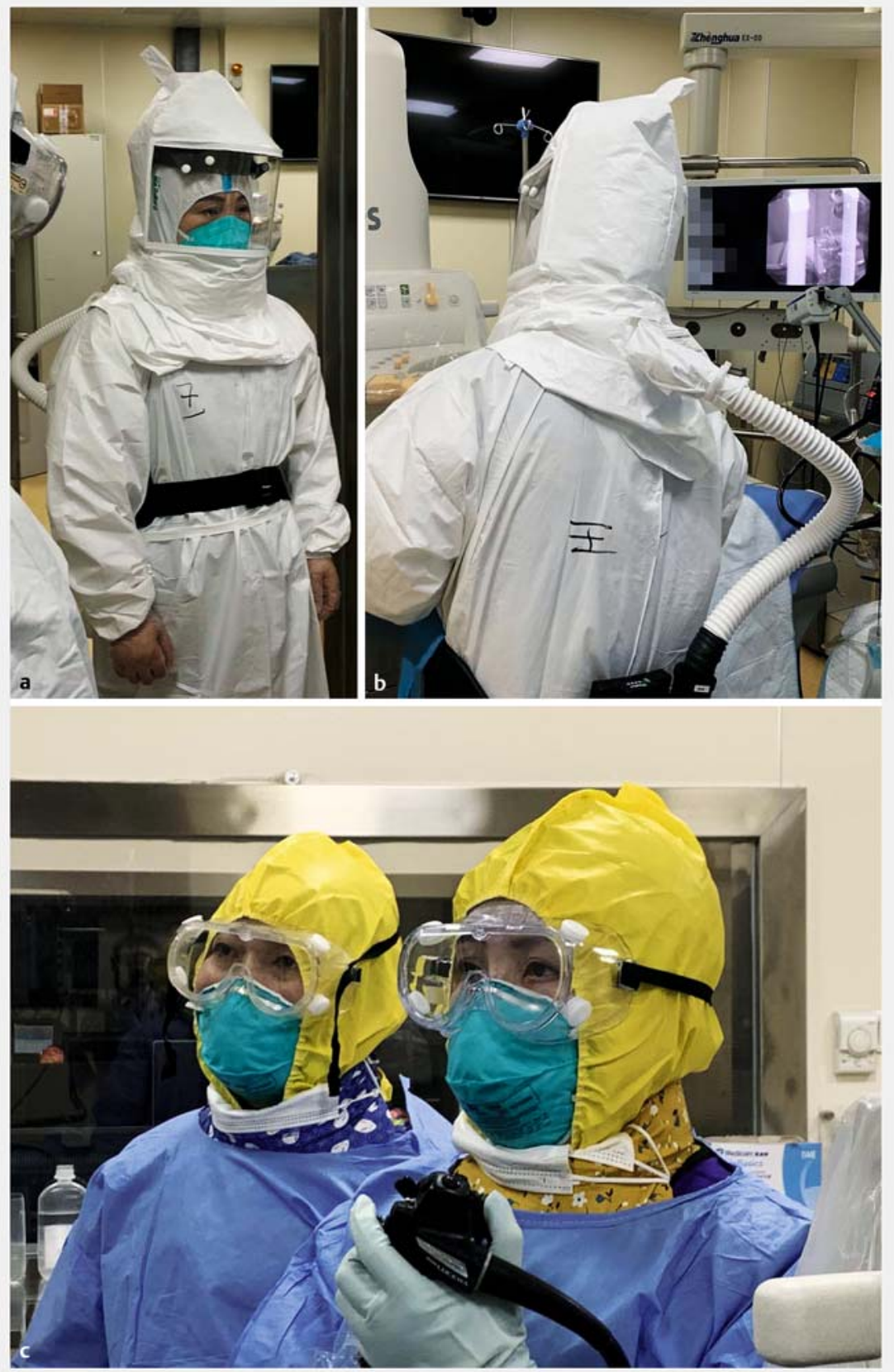

- Fig. 1 Photographs of the personal protection used during endoscopic retrograde cholangiopancreatography: a,b at biosafety level 3 for a patient with COVID-19; $\mathbf{c}$ at biosafety level 2 for patients in whom COVID-19 has been excluded.

monly used disinfectants, there seem to be no additional measures necessary for duodenoscope disinfection after ERCP
$[3,4]$. To avoid generation of an aerosol, bedside pre-cleaning of the used endoscope was omitted from the procedure. 
As had been suggested, the procedure of soaking in $0.3 \%$ peracetic acid was added [2]. With regard to single-use duodenoscopes, we think this would be a feasible method if the financial situation allowed. Strict management for COVID-19 patients during hospitalization, not only during ERCP processing, may make more sense in terms of reducing the risk of transmission among patients and medical staff.

The reported patient has recovered quickly after ERCP, and 1 week later he was transferred to the Department of Infectious Disease for further isolation and treatment.

\section{Competing interests}

The authors declare that they have no conflict of interest.
The authors

Hongling Wang ${ }^{1,2}$, Yafei Zhang ${ }^{1,2}$, Lan Liu ${ }^{1,2}$, Xiaodan Zhang ${ }^{1,2}$, Qiu Zhao ${ }^{1,2}$

1 Department of Gastroenterology, Zhongnan Hospital of Wuhan University, Wuhan, China

2 Clinical Center and Key Lab of Intestinal and Colorectal Diseases of Hubei Province, Wuhan, China

\section{Corresponding author}

\section{Qiu Zhao, MD}

Department of Gastroenterology, Zhongnan Hospital of Wuhan University, 169 Donghu Road, Wuhan 430071, Hubei, China Fax: +86-27-67812522

qiuzhao@whu.edu.cn

\section{References}

[1] [In Chinese] http://www.nhc.gov.cn/yzygj/ s7653p/202002/ d4b895337e19445f8d728fcaf1e3e13al files/ab6bec7f93e64e7f998d802991203cd6.pdf

[2] [In Chinese] http://www.csde.org.cn/news/ detail.aspx?article_id $=2884$

[3] ASGE Quality Assurance in Endoscopy Committee. ASGE guideline for infection control during $\mathrm{Gl}$ endoscopy. Gastrointest Endosc 2018; 87: 1167-1179

[4] Geller C, Varbanov M, Duval RE. Human coronaviruses: insights into environmental resistance and its influence on the development of new antiseptic strategies. Viruses 2012; 4: 3044-3068

Bibliography

Endoscopy 2020; 52: 933-934

DOI 10.1055/a-1194-4745

ISSN $0013-726 \mathrm{X}$

(c) 2020. Thieme. All rights reserved. Georg Thieme Verlag KG Rüdigerstraße 14, 70469 Stuttgart, Germany 